\title{
A Fast LP Approach for Enhanced Utilization of Variable Impedance Based FACTS Devices
}

\author{
M. Sahraei-Ardakani, Member, IEEE, and K. W. Hedman, Member, IEEE
}

\begin{abstract}
Transmission systems are under stress and need to be upgraded. Better utilization of the existing grid provides a fast and cheap alternative to building new transmission. One way to improve the utilization of the transmission network is power flow control via flexible AC transmission system (FACTS) devices. While FACTS devices are used today, the utilization of these devices is limited; traditional dispatch models (e.g., security constrained economic dispatch) assume a fixed, static transmission grid even though it is rather flexible. The primary barrier is the complexity that is added to the power flow problem. The mathematical representation of the DC optimal power flow, with the added modeling of FACTS devices, is a nonlinear program (NLP). This paper presents a method to convert this NLP into a mixed-integer linear program (MILP). The MILP is reformulated as a two-stage linear program, which enforces the same sign for the voltage angle differences for the lines equipped with FACTS. While this approximation does not guarantee optimality, more than $98 \%$ of the presented empirical results, based on the IEEE 118 bus and Polish system, achieved global optimality. In the case of suboptimal solutions, the savings were still significant and the solution time was dramatically reduced.
\end{abstract}

Index Terms--FACTS devices, linear programming, power generation dispatch, power generation economics, power system economics, power transmission economics, transfer capability, transmission topology optimization.

\section{NOMENCLATURE}

\section{Sets}

G Set of generators.

$g \quad$ Index of generators, $g \in G$.

$g(n)$ Set of generators connected to node $n$.

$K \quad$ Set of all transmission elements, line or transformer.

$k \quad$ Index of transmission element, $k \in K$.

$\bar{K} \quad$ Set of transmission lines equipped with FACTS, $\bar{K} \subset K$.

$\bar{k} \quad$ Index of lines equipped with FACTS, $\bar{k} \in \bar{K} \subset K$.

$\underline{K} \quad$ Set of transmission elements not equipped with FACTS, $\underline{K} \subset K$.

$\underline{k} \quad$ Index of transmission elements not equipped with FACTS, $\underline{k} \in \underline{K} \subset K$.

$N \quad$ Set of nodes.

$n \quad$ Index for buses, $n \in N$.

This manuscript was originally submitted on December 3, 2014 and revised on March 27, 2015.

Mostafa Sahraei-Ardakani and Kory W. Hedman are with the School of Electrical, Computer, and Energy Engineering, Arizona State University, Tempe, AZ 85287 USA (e-mail: Mostafa@asu.edu; Kory.Hedman@asu.edu). $n(g) \quad$ Node location of generator $g$.

$\sigma^{+}(n)$ Set of lines specified as to node $n$.

$\sigma^{-}(n)$ Set of lines specified as from node $n$.

\section{Parameters}

$B_{k} \quad$ Electrical susceptance of transmission element $k$.

$X_{k} \quad$ Electrical reactance of transmission element $k$.

$c_{g} \quad$ Marginal cost of generator $g(\$ / \mathrm{MWh})$.

$d_{n} \quad$ Demand at bus $n$.

$P_{g}^{\max }$ Maximum output of generator $g$.

$P_{g}^{\min }$ Minimum output of generator $g$.

$F_{k}^{\max }$ Capacity of transmission line $k$.

$B \bar{k}^{\max } \quad$ Maximum susceptance of line $\bar{k}$ equipped with

\section{FACTS.}

$B_{\bar{k}}^{\min } \quad$ Minimum susceptance of line $\bar{k}$ equipped with

\section{FACTS.}

$z_{k}^{0} \quad$ Binary variable indicating the sign of voltage angle difference on line $k$ in the initial dispatch with no FACTS.

$N_{\text {FACTS }}$ Maximum number of FACTS devices in allocation problem.

\section{Variables}

$P_{g} \quad$ Real power output variable for generator $g$.

$F_{k} \quad$ Real power flow through line $k$.

$\theta_{n} \quad$ Voltage angle at node $n$.

$B_{\bar{k}} \quad$ Susceptance of line $\bar{k}$ equipped with FACTS.

$z_{\bar{k}} \quad$ Binary variable indicating the sign of voltage angle difference on line $\bar{k}$ equipped with FACTS.

$x_{k} \quad$ Binary variable in FACTS allocation problem, indicating whether line $k$ is equipped with FACTS or not.

\section{INTRODUCTION}

$\mathrm{T}$ HE US electricity industry has an annual revenue that reaches into the hundreds of billions of dollars; efficient operation of the electric power grid is, therefore, paramount [1]. Transmission bottlenecks are one particular source of system inefficiency. The transmission network in the US is under stress and needs to be upgraded [2]-[3]. One challenge to this problem is the long process required to site new transmission due to a variety of concerns, including not in my backyard (NIMBY). More efficient utilization of the existing network is a much faster alternative and, while it cannot replace the need for new transmission, it is substantially cheaper and can significantly delay the need for new transmission. Furthermore, it is obvious that the system should be utilized to 
its full capability as opposed to being under-utilized. Such a call for studying alternative solutions for transmission upgrade projects is also identified by the Federal Energy Regulatory Commission (FERC) order 1000 [4].

The electric power grid is one of the largest and most complex engineered systems to date [5]. The Advanced Research Projects Agency - Energy (ARPA-E) Green Electricity Network Integration (GENI) initiative invested over \$30M in both new transmission hardware, as well as software that enhances utilization of hardware, to improve the ability to control the flow of power across the grid. Such power flow control can be achieved with transmission switching [6]-[9], phase shifters, and adjusting lines' reactances using flexible $\mathrm{AC}$ transmission systems (FACTS) devices [10]-[11]. Previous research has shown that transmission switching (TS) can reduce operational costs [6], [7], [12], improve reliability [13], and improve the management of intermittent resources such as wind and solar [14]. Transmission switching can also be used as a corrective action in contingency analysis packages [15]-[17]. The main challenges facing transmission switching are computational complexity [18], ensuring AC feasibility [19], and ensuring the stability of the switching action itself [20].

Continuous adjustment of the line's reactance aims at achieving the same goals with less concern regarding the stability of the system. Inclusion of FACTS adjustments in DC optimal power flow (DCOPF), a linear program (LP) itself, makes the problem a non-linear program (NLP), which is computationally intense like the TS problem. This paper contributes to the existing literature by proposing a reformulation of the NLP problem along with a decomposition of the problem, which constructs a fast two-stage LP.

Some types of series FACTS enable significant adjustment of a line's reactance [21]-[22]. In this paper, the term "FACTS" is loosely used to refer to those devices, e.g., unified power flow controllers (UPFC) and thyristor controlled series compensators (TCSC). The formulation presented in this paper is also applicable to the unconventional FACTS technology such as Smart Wire Grid device [23].

A study by the US Department of Energy acknowledges the benefits of FACTS devices and their role for improved operation in the smart grid [24]. With power flow control, power can be rerouted to lines that are not congested in an attempt to avoid transmission bottlenecks. The transfer capability is, thus, enhanced to allow for cheaper resources (e.g., renewables) to be dispatched to reduce operational costs. The deliverability of reserves is also enhanced, enabling an improvement in reliability [25]-[28]. It is estimated that FACTS devices could increase the transfer capability over the existing transmission lines by fifty percent [29].

FACTS devices are already a part of our transmission network. ISO-NE has thirteen installed and three planned FACTS in its territory [30]. Five EPRI-sponsored FACTS devices are currently operating in AEP's territory (Kentucky), BPA (Oregon), CSW (Texas), TVA (Tennessee), and NYPA (New York) [31]. In PJM, Primary Power LCC is developing the Grid Plus project, which involves installation of several FACTS devices. The project aims at increasing the transfer capability from west to east, reducing congestion, and improving system stability [32].

Previous research has shown that FACTS devices are bene- ficial in loop flow cancellation [33]-[34], help reduce cost and improve reliability [35]-[36], and enhance the benefits of demand response [37]. Although the benefits of FACTS devices are known [38], the industry does not change FACTS devices' set-points frequently. Recent research has addressed this problem and proposed different methods to overcome it: (i) inclusion of a price signal [39], (ii) transmission bidding in a complete real-time market [40], and (iii) compensation based on market value [41]-[42]. However, the main problem, which is computational difficulty, has not been properly addressed.

Reference [43] aims at addressing the computational complexity challenge by proposing a regression-based model to improve the transfer capability. However, [43] suffers from natural shortcomings of statistical models and would only produce reasonable solutions when the system operates near the historical states. A formal optimization based formulation would provide better insights into the problem and avoid the natural limitations of statistical models.

As mentioned before, the problem representing optimal adjustment of FACTS setting is an NLP. NLPs are computationally intense and may not converge in the limited time available. This paper presents a method to reformulate this NLP into a mixed-integer linear program (MILP), with binary variables representing the signs of the voltage angle differences over the lines equipped with FACTS. If the adjustments made by FACTS devices (to the line's impedance) do not change the nature of a line's reactance, from inductive to capacitive or vice versa, the voltage angle difference sign is still an indicator for the direction of the line's flow. Note that such change in the nature of a line's reactance would involve an adjustment of more than $100 \%$ in the reactance of the line. Such is not likely to happen due to a variety of reasons including stability concerns. Assuming that optimal adjustment of FACTS devices will not change those voltage angle difference signs, the MILP can be solved only for the particular branch and bound node of the problem, representing those signs from the original dispatch. Thus, as long as the FACTS device adjustments do not cause a change in the direction of the lines' flows, for the lines equipped with FACTS devices, the problem can be solved as an LP instead of a MILP. This will make the problem a two-stage LP where, in the first stage, an initial DCOPF is solved without consideration of FACTS devices. At the second stage, the FACTS devices are modeled while enforcing the same voltage angle difference signs as the original dispatch, for the lines with FACTS. The mathematical representations of both stages are LPs. This reformulation significantly reduces the computational burden of the problem, thereby making the integration of FACTS operation in security constrained economic dispatch (SCED) an immediate practical possibility. Note that there is no guarantee that the method converges to the globally optimal solution, unless of course the original MILP formulation is later solved. The globally optimal solution may be in another node of the MILP, with at least one change in the sign of the voltage angle difference for one line equipped with FACTS. However, our two-stage linear method will find a solution with a cost lower or equal to the original dispatch much faster than the original MILP.

Note that obtaining significant cost savings with little added computational burden is more important than achieving global optimality as global optimality is not achieved today. 
Accomplishing such a task provides an innovative and nearterm practical solution. To achieve this result, we illustrate one very important insight regarding this assumption that the sign of the angle difference will not change. This assumption states that the direction of the flow will not change, assuming that FACTS adjustments will not change the nature of a line's reactance. The most beneficial location to place such FACTS are generally on key transmission bottlenecks. Given the nature of the power grid where power flows from remote bulk power facilities to key load centers, there are many key transmission elements where the direction of the flow is rather predictable (for heavily meshed networks, this may be less the case). We contend that the accuracy of this assumption is high based on two observations: (i) the placement of these FACTS devices should be on key transmission lines and (ii) the ability to predict the direction of flow (period by period) should be high. Finally, our results support this conjecture and in more than $98 \%$ of the simulation studies presented in this paper, our method was able to find the globally optimal solution, which was confirmed by solving the full MILP. While there is no guarantee, the strength in this approach does not disappear: a fast algorithm that determines FACTS settings very quickly. The simulation studies on the IEEE 118 bus system show that the co-optimization of FACTS setting, alongside generation dispatch, would lead to significant cost savings and improved transfer capability.

The rest of this paper is structured as follows: Section II includes the basic non-linear formulation and the method to reformulate the problem. Section III presents the simulation studies on the IEEE 118 bus system. Some remarks regarding the future work and next steps are pointed out in Section IV. Finally, Section V concludes the paper.

\section{PRoblem Formulation AND Reformulation}

Optimal power flow (OPF) is the essential part of market solvers for any power system. OPF, in its original form, is a nonlinear and non-convex problem. In existing markets, linear approximations of the OPF problems are used instead of the more complex nonlinear formulation due to the limited computational time, market transparency issues, and market pricing issues. Adjustments to this solution, to ensure AC feasibility, are made out-of-market [44]. For a system where generators have constant marginal costs, (1)-(5) present the DCOPF problem:

$\min \sum_{g} c_{g} P_{g}$

$P_{g}^{\min } \leq P_{g} \leq P_{g}^{\max }$

$-F_{\mathrm{k}}^{\max } \leq F_{\mathrm{k}} \leq F_{\mathrm{k}}^{\max }$

$F_{\mathrm{k}}-B_{k}\left(\theta_{n}-\theta_{m}\right)=0$

$\sum_{k \in \sigma^{+}(n)} F_{k}-\sum_{k \in \sigma^{-}(n)} F_{k}+\sum_{g \in g(n)} P_{g}=d_{n}$

Terms $\mathrm{n}$ and $\mathrm{m}$ in (4) are the "to" and "from" nodes of line $\mathrm{k}$ respectively. Since, both the objective function and the constraints are linear, the DCOPF is an LP. FACTS devices enable adjustment of lines' susceptances, thereby making the term $B_{k}$ in (4) a variable instead of a parameter. Therefore, replacing (4) with (6)-(8) would include optimal adjustment of FACTS devices in the DCOPF problem. Note that $B_{\bar{k}}$ is a variable in the optimization problem.

$$
\begin{array}{ll}
F_{\underline{k}}-B_{\underline{k}}\left(\theta_{n}-\theta_{m}\right)=0 & \forall \underline{k} \\
F_{\bar{k}}-B_{\bar{k}}\left(\theta_{n}-\theta_{m}\right)=0 & \forall \bar{k} \\
B_{\bar{k}}^{\min } \leq B_{\bar{k}} \leq B_{\bar{k}}^{\max } & \forall \bar{k}
\end{array}
$$

Equation (7) is a non-linear constraint, because it includes the multiplication of $\theta$ and $B_{\bar{k}}$. Thus, the subsequent DCOPF becomes an NLP. However, (7)-(8) can be rewritten as linear inequality constraints, without the loss of generality, depending on the sign of the voltage angle difference.

if $\left(\theta_{n}-\theta_{m}\right) \geq 0$ :

$B_{\bar{k}}^{\min }\left(\theta_{n}-\theta_{m}\right) \leq F_{\bar{k}} \leq B_{\bar{k}}^{\max }\left(\theta_{n}-\theta_{m}\right) \quad \forall \bar{k}$

if $\left(\theta_{n}-\theta_{m}\right) \leq 0$ :

$B_{\bar{k}}^{\max }\left(\theta_{n}-\theta_{m}\right) \leq F_{\bar{k}} \leq B_{\bar{k}}^{\min }\left(\theta_{n}-\theta_{m}\right) \quad \forall \bar{k}$

For a positive angle difference $\left(\theta_{n}-\theta_{m}\right)$, the lower and higher limits on susceptance would also impose the lower and higher limits on the power flow. However, for negative angle difference, the lower suceptance limit would impose the higher limit on the power flow and vice versa. The "if conditions" in (9)-(10) can be modeled with binary variables, making the problem a mixed-integer nonlinear program (MINLP):

$$
\begin{array}{ll}
\left(\left(1-z_{\bar{k}}\right) B_{\bar{k}}^{\min }+z_{\bar{k}} B_{\bar{k}}^{\max }\right)\left(\theta_{n}-\theta_{m}\right) \geq F_{\bar{k}} & \forall \bar{k} \\
\left(\left(1-z_{\bar{k}}\right) B_{\bar{k}}^{\max }+z_{\bar{k}} B_{\bar{k}}^{\min }\right)\left(\theta_{n}-\theta_{m}\right) \leq F_{\bar{k}} & \forall \bar{k} \\
\left(1-z_{\bar{k}}\right) \theta_{m}+z_{\bar{k}} \theta_{n} \geq\left(1-z_{\bar{k}}\right) \theta_{n}+z_{\bar{k}} \theta_{m} & \forall \bar{k} \\
z_{\bar{k}} \in\{0,1\} &
\end{array}
$$

Positive voltage angle difference enforces $z_{\bar{k}}$ to take 1 as its value, while negative voltage angle difference sets $z_{\bar{k}}$ to 0 . The problem then can be reformulated to a MILP, using a big $M$ reformulation:

$$
\begin{array}{ll}
z_{\bar{k}} B_{\bar{k}}^{\min }\left(\theta_{n}-\theta_{m}\right)-\left(1-z_{\bar{k}}\right) M \leq F_{\bar{k}} & \forall \bar{k} \\
\left(1-z_{\bar{k}}\right) B_{\bar{k}}^{\max }\left(\theta_{n}-\theta_{m}\right)-z_{\bar{k}} M \leq F_{\bar{k}} & \forall \bar{k} \\
z_{\bar{k}} B_{\bar{k}}^{\max }\left(\theta_{n}-\theta_{m}\right)+\left(1-z_{\bar{k}}\right) M \geq F_{\bar{k}} & \forall \bar{k} \\
\left(1-z_{\bar{k}}\right) B_{\bar{k}}^{\min }\left(\theta_{n}-\theta_{m}\right)+z_{\bar{k}} M \geq F_{\bar{k}} & \forall \bar{k} \\
\theta_{n}+\left(1-z_{\bar{k}}\right) M \geq \theta_{m} & \forall \bar{k} \\
\theta_{m}+z_{\bar{k}} M \geq \theta_{n} & \forall \bar{k} \\
z_{\bar{k}} \in\{0,1\} & \\
M \gg \operatorname{Max}\left\{F_{\bar{k}}+B_{k}\left(\theta_{m}-\theta_{n}\right)\right\} &
\end{array}
$$$$
\forall \bar{k} \quad(15)
$$$$
\bar{k}
$$

If the directions were known (i.e., the variable $\mathrm{z}_{\overline{\mathrm{k}}}$ in (15)(21) was fixed), the complexity of the problem would reduce to an LP, which can then be solved efficiently for large scale real power systems.

Although, it is not possible to know the direction of each line flow before solving the OPF, the mathematical structure of this formulation can be exploited. There are many lines for which the operators know the direction of the power flow, e.g., main import lines to urban areas as well as major tie lines. Particularly, a two-stage solver can be implemented to first solve the linear DCOPF without FACTS, (1)-(5), and initialize the direction of the power flows for the second stage. Then, the second stage enforces the power flows on the lines equipped with FACTS to be at their initial direction and solves another LP (1)-(3), (5)-(6), (15)-(22) to find the optimal adjustment of the FACTS devices. This process is shown in Fig. 
1. Since FACTS devices are often installed on major transmission lines, it is not expected that the power flow on such lines change direction.

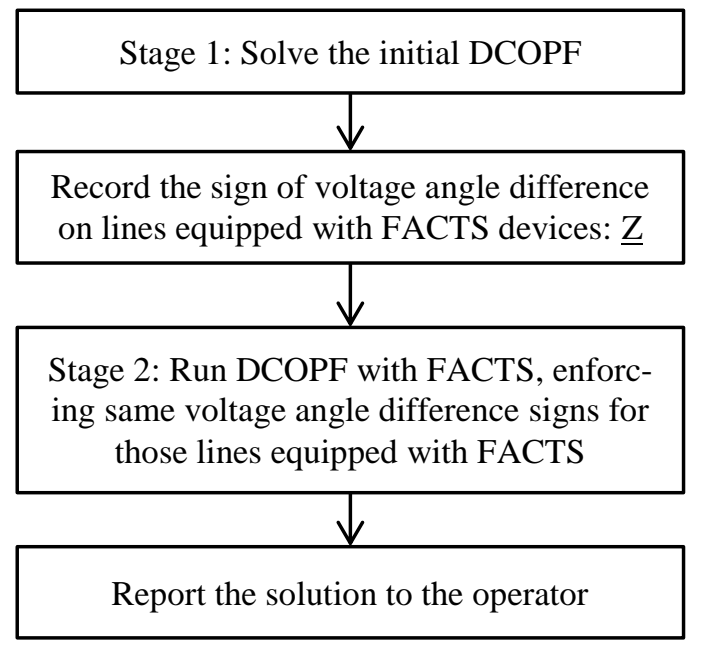

Fig. 1. The two-stage LP-based algorithm for enhanced operation of FACTS devices.

The initial dispatch is a feasible solution for the second stage problem. Thus, the two-stage method presented in this paper always finds a solution with a cost less than or equal to the cost of the initial dispatch. This solution may or may not be globally optimal. When solving the full MILP formulation, if the signs for the voltage angle differences, for the lines equipped with FACTS, are the same as the signs for the solution from the two-stage LP approach, this confirms the solution from the two-stage LP approach is optimal. While, in theory, the flows can change direction after adjustment of FACTS settings, it is highly unlikely that this happens on the lines equipped with FACTS devices. The reason is that improving transfer capability would often involve rerouting the flow from congested lines to alternative parallel paths that are not congested. Reversing the power flow direction would reverse the flow direction for the parallel paths as well, which is highly unlikely. Thus, instead of reversing the power flows, it is expected to see more flows on the parallel paths to the congested lines in the same direction.

\section{Simulation STUdieS}

In this section, the method explained in Section II is applied to IEEE 118 bus system and a large-scale Polish system.

\section{A. IEEE 118-Bus System}

The test case data are taken from [45], with additional data and modifications according to [46]. The full test case dataset can be obtained from [47]. With this dataset, the results of optimal transmission switching (OTS) [6] can be replicated. Fig. 2 shows the total cost when up to 20 lines are allowed to be switched. The total cost of dispatch for the system with no adjustment to the transmission system is $\$ 2,074$. With a transportation model of the transmission network, the dispatch cost would go down to $\$ 1,303$, which for this particular test case is also equal to the cost obtained from economic dispatch (transmission-less model). Therefore, the total potential savings would be $\$ 771$, the difference between the two costs. The transportation model of the transmission network only consid- ers the capacity limits and ignores the power flow constraints (4). Note that the dispatch cost obtained from a transportation model would be the lowest cost that can be achieved, assuming full power flow control, without building new lines. Some research has adopted economic dispatch cost (ignoring the transmission network altogether) as a basis for calculating the savings [48]. However, the cost associated to the capacity limits cannot be lifted with transmission switching or FACTS adjustments. The only part of the transmission related costs that can be avoided by harnessing transmission flexibility (OTS or FACTS utilization) is the portion associated with the power flow constraints. Therefore, in this paper, the savings are compared to the transportation network solution. Fig. 2 shows that with 20 transmission switching actions, $67 \%$ of the possible savings is achieved.

Fig. 3 shows the total cost when up to 40 FACTS devices are allowed in the system. The figure shows how the dispatch cost decreases as more FACTS devices are allowed to be used. The figure also shows the relationship between cost and FACTS capacity (how much a FACTS device can change the reactance of a line). The location of the devices is picked based on the linear method presented in the previous section to maximize the savings. Note that the number of FACTS allowed does not necessarily equal the number of FACTS that is actually used. The graph shows that the cost reduction is more significant for the first few FACTS and then the cost curves saturate. Such effect is more noticeable when the FACTS capacity is larger. In fact, for the case with $70 \%$ and $90 \%$ capacity, only 24 and 11 devices would be needed to achieve the maximum saving. That is equivalent to $100 \%$ savings leading to a dispatch cost close to $\$ 1,303$.

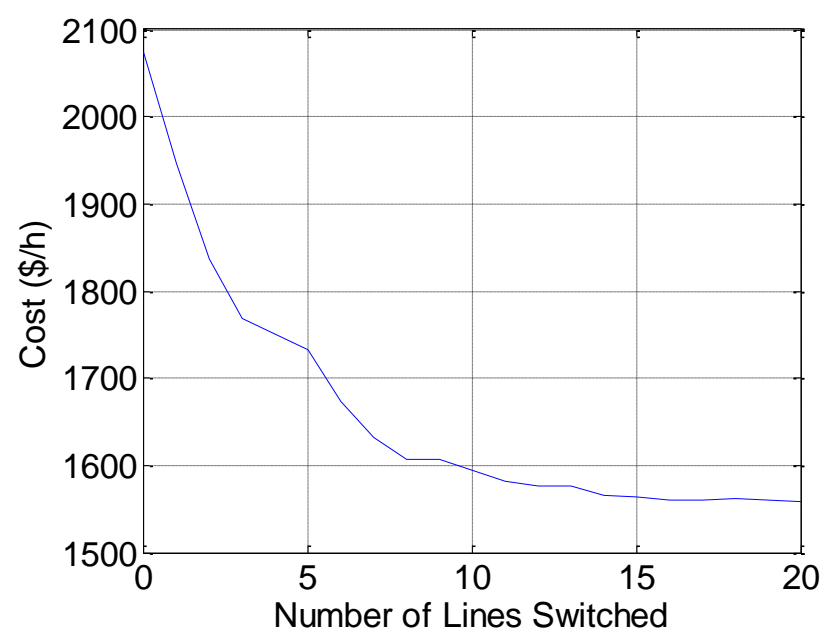

Fig. 2. Total cost using optimal transmission switching (OTS) for IEEE 118 bus system with up to 20 allowed switching actions.

The formulation presented in the previous section aims at enhanced adjustment of FACTS set point in the operational SCED, assuming their location is fixed. However, note that we first solve a FACTS placement investment problem, for the results shown in Fig. 3 and Tables I-III, to ensure the devices are placed at reasonable locations. 
TABLE I

SOLUTION PROPERTIES UNDER DIFFERENT SCENARIOS FOR IEEE 118 BUS SYSTEM

\begin{tabular}{|c|c|c|c|c|c|c|c|c|c|c|c|}
\hline & \multirow{2}{*}{$\begin{array}{l}\text { Base } \\
\text { Case }\end{array}$} & \multirow{2}{*}{$\begin{array}{c}\text { Transport. } \\
\text { Model }\end{array}$} & \multirow{2}{*}{$\begin{array}{l}\text { OTS } \\
(36)\end{array}$} & \multicolumn{8}{|c|}{ FACTS Capacity } \\
\hline & & & & $2 \%$ & $5 \%$ & $10 \%$ & $20 \%$ & $30 \%$ & $50 \%$ & $70 \%$ & $90 \%$ \\
\hline \# of FACTS Utilized & -- & $\overline{--}$ & $\overline{--}$ & 13 & 29 & 27 & 31 & 23 & 31 & 24 & 11 \\
\hline \# of Congested Lines & 2 & 68 & 4 & 2 & 2 & 3 & 5 & 5 & 6 & 7 & 6 \\
\hline $\operatorname{Cost}(\$ / h)$ & 2074.4 & 1303.3 & 1557.8 & 2015.7 & 1887.4 & 1684.9 & 1502.2 & 1454.3 & 1335.2 & 1306.0 & 1303.3 \\
\hline Savings (\%) & 0 & 100 & 67 & 8 & 24 & 51 & 74 & 80 & 96 & 100 & 100 \\
\hline
\end{tabular}

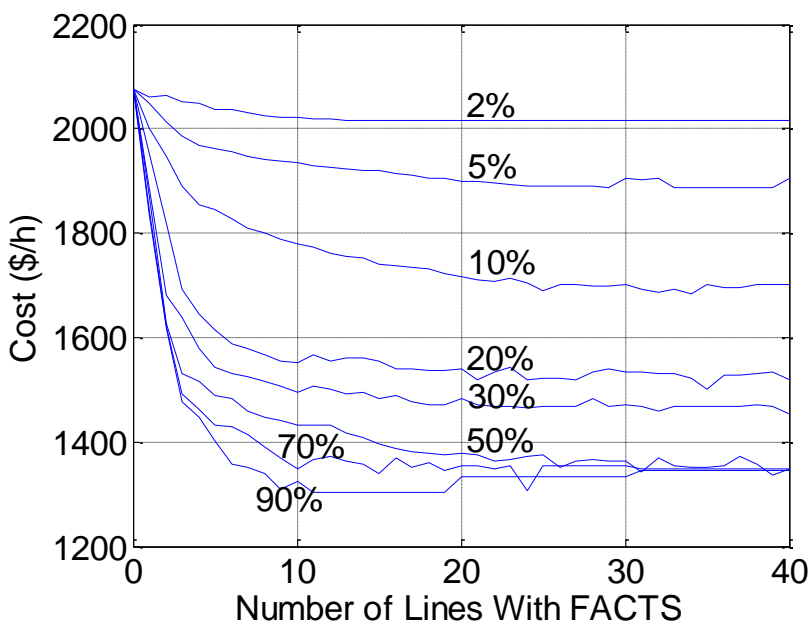

Fig. 3. Total cost with up to 40 FACTS devices at different capacity levels placed optimally in the IEEE 118 bus system.

The mathematical program to determine the optimal allocation of FACTS devices is even more computationally intense, because it involves additional binary variables indicating whether a line should be equipped with FACTS. This is another area where the method developed in this paper finds its application to deal with even more challenging problems with additional non-convexities. The mathematical representation of the allocation problem, with the linear approximation developed in section II, is shown in (23)-(38). Note that the linear approximation is used only in the power flow part of the problem and not in the allocation part of the problem. Before solving (23)-(38), first, a DCOPF is solved to identify the sign of voltage angle differences for all lines $\left(\mathrm{z}_{\mathrm{k}}^{0}\right)$. Subsequently, assuming the same signs for the lines equipped with FACTS, the allocation problem is solved. Therefore, $\mathrm{z}_{\mathrm{k}}^{0}$ is a parameter and not a variable. $x_{k}$ takes a value of 1 to indicate that a variable impedance FACTS device should be installed on line $\mathrm{k}$, and zero otherwise. Note that, this is a FACTS siting problem formulation that only considers the operational cost assuming a fixed predetermined number of FACTS devices ready for installation. Thus, the FACTS capital cost in (23)-(38) is a sunk cost and does not go into the objective function. In a more advanced siting problem, the savings calculated in (23)(38) compared to a business as usual case with no FACTS devices should be aggregated and discounted over the lifetime of the devices. This discounted value should be compared with the required capital cost of FACTS devices in a net present value type of a calculation to not only optimize FACTS location but also optimize the number of such devices.

$\min \sum_{g} c_{g} P_{g}$

$P_{g}^{\min } \leq P_{g} \leq P_{g}^{\max }$

$-F_{\mathrm{k}}^{\max } \leq F_{\mathrm{k}} \leq F_{\mathrm{k}}^{\max }$

$\forall k$

$\begin{array}{ll}\sum_{k \in \sigma^{+}(n)} F_{k}-\sum_{k \in \sigma^{-}(n)} F_{k}+\sum_{g \in g(n)} P_{g}=d_{n} & \forall n \\ \left(1-x_{k}\right)\left(F_{k}-B_{k}\left(\theta_{n}-\theta_{m}\right)\right)=0 & \forall k \\ x_{k}\left(F_{k}-B_{k}\left(\theta_{n}-\theta_{m}\right)\right)=0 & \forall k \\ x_{k} B_{k}^{\min } \leq x_{k} B_{k} \leq x_{k} B_{k}^{\max } & \forall \mathrm{k} \\ x_{k}\left(z_{k}^{0} B_{k}^{\min }\left(\theta_{n}-\theta_{m}\right)-\left(1-z_{k}^{0}\right) M\right) \leq x_{k} F_{k} & \forall k \\ x_{k}\left(\left(1-z_{\bar{k}}\right) B_{k}^{\max }\left(\theta_{n}-\theta_{m}\right)-z_{k}^{0} M\right) \leq x_{k} F_{\bar{k}} & \forall k \\ x_{k}\left(z_{k}^{0} B_{k}^{\max }\left(\theta_{n}-\theta_{m}\right)+\left(1-z_{k}^{0}\right) M\right) \geq x_{k} F_{\bar{k}} & \forall k \\ x_{k}\left(\left(1-z_{k}^{0}\right) B_{k}^{\min }\left(\theta_{n}-\theta_{m}\right)+z_{k}^{0} M\right) \geq x_{k} F_{\bar{k}} & \forall k \\ x_{k}\left(\theta_{n}+\left(1-z_{k}^{0}\right) M\right) \geq x_{k} \theta_{m} & \forall k \\ x_{k}\left(\theta_{m}+z_{k}^{0} M\right) \geq x_{k} \theta_{n} & \forall k \\ x_{k} \in\{0,1\} & \forall k \\ \sum_{k} x_{k} \leq N_{F A C T S} & \\ M \gg \operatorname{Max}\left\{F_{k}+B_{k}\left(\theta_{m}-\theta_{n}\right)\right\} & \end{array}$

In order to achieve a solution in a reasonable time, relatively large optimality gaps (3\% for the cases with less than 10 allowable FACTS and $6 \%$ for the cases with more than 10 allowable FACTS) were picked; such optimality gaps are only used for the placement (investment) problem and not the operational SCED model. Note that, as can be seen by Fig. 3, the curves are not monotonically decreasing, which occurs due to the optimality gaps.

Fig. 3 presents 320 simulation studies. All of these cases were also simulated using the original MILP formulation. For all but two of the cases shown in Fig. 3 our two-stage LP formulation was able to find the exact same solution as the global optimal solution found by MILP. This means that the optimal solution to the original MILP ((1)-(3), (5)-(6), (15)-(22)), is the node representing the initial power flow directions for the lines equipped with FACTS for 318 out of 320 cases presented in Fig. 3. Such results confirm the intuitive engineering perspective presented earlier in the paper: it is not likely that the power flow on lines, which have FACTS installed on them, change direction. For the two cases that the linear proxy developed in section II was not able to find the global optimal solution, the cost difference was $0.02 \%$ and, thus, the linear model was able to find a near optimal solution. Our method took on average 20 milliseconds for each simulation, while the MILP formulation took on average 529 milliseconds. Thus, our method is 26 times faster than the MILP formulation for IEEE 118 bus system. While both solution times are small, it is very important to note that the scalability of this two-stage LP approach is by far better than such MILPs with disjunctive constraints; thus, even better speed-ups in solution time would be expected for large-scale systems where the solution times will be much longer. As expected, the simulations also confirmed that the computational time for MILP is sensitive to the number of FACTS devices in the system, while the computational time for our two-stage LP formulation is robust and scales well. 
Table I summarizes the properties of the solutions under different scenarios. The scenarios include (i) a base case with no FACTS or switching, (ii) OTS, which represents the best possible solution attainable via transmission switching, and (iii) eight scenarios with different capacities for FACTS devices. The optimal transmission switching solution is obtained with 36 switching actions and it achieves $67 \%$ of the potential savings that a transportation model would otherwise achieve. Table I shows that, for the scenarios with $20 \%$ or larger FACTS capacity, the flexibility provided by FACTS can result in larger cost savings than OTS. Moreover, with larger FACTS, significant savings can be achieved with only few devices.

Table II shows the generation dispatch for the base case, the transportation network, OTS, and four scenarios with FACTS devices. The table also includes cost information for the generators. Generators 9, 10, 15, 18, and 19 are the five units that experience changes in their dispatch due to the modifications to the transmission system. Generator 10 is one of the cheaper generators that does not produce energy in the base case, due to the transmission limits. Both transmission switching and FACTS provide transfer capabilities that would allow this generator to produce and replace the more expensive generators 15, 18, and 19. The number of FACTS devices used for the scenarios presented in Table II is consistent with the results shown in Table I.

TABLE II

GENERATION DISPATCH (MW) FOR THE SOLUTIONS UNDER DIFFERENT

\begin{tabular}{l|rrrrrrr}
\multicolumn{8}{c}{ SCENARIOS } \\
\hline & Base & Transport. & OTS & \multicolumn{5}{c}{ FACTS Capacity } \\
\cline { 2 - 8 } Case & Model & $(36)$ & $30 \%$ & $50 \%$ & $70 \%$ & $90 \%$ \\
\cline { 2 - 8 } Gen 1 & 550 & 550 & 550 & 550 & 550 & 550 & 550 \\
Gen 2 & 0 & 0 & 0 & 0 & 0 & 0 & 0 \\
Gen 3 & 263 & 320 & 320 & 320 & 320 & 320 & 320 \\
Gen 4 & 414 & 414 & 414 & 414 & 414 & 414 & 414 \\
Gen 5 & 0 & 0 & 0 & 0 & 0 & 0 & 0 \\
Gen 6 & 0 & 0 & 0 & 0 & 0 & 0 & 0 \\
Gen 7 & 304 & 304 & 304 & 304 & 304 & 304 & 304 \\
Gen 8 & 0 & 0 & 0 & 0 & 0 & 0 & 0 \\
Gen 9 & 0 & 0 & 0 & 0 & 0 & 150 & 0 \\
Gen 10 & 0 & 214 & 68 & 107 & 191 & 63 & 214 \\
Gen 11 & 491 & 491 & 491 & 491 & 491 & 491 & 491 \\
Gen 12 & 492 & 492 & 492 & 492 & 492 & 492 & 492 \\
Gen 13 & 805 & 805 & 805 & 805 & 805 & 805 & 805 \\
Gen 14 & 577 & 577 & 577 & 577 & 577 & 577 & 577 \\
Gen 15 & 72 & 0 & 11 & 0 & 0 & 0 & 0 \\
Gen 16 & 0 & 0 & 0 & 0 & 0 & 0 & 0 \\
Gen 17 & 352 & 352 & 352 & 352 & 352 & 352 & 352 \\
Gen 18 & 140 & 0 & 134 & 107 & 23 & 0 & 0 \\
Gen 19 & 59 & 0 & 0 & 0 & 0 & 0 & 0 \\
\hline
\end{tabular}

Table III shows the optimal placement of FACTS as well as the FACTS capacity under the two scenarios with higher permissible capacities. The table also shows lines' loadings as a percentage of their capacities in the original dispatch without FACTS devices. The reactances of the lines that were heavily loaded were often increased to reroute the power flow to other paths. Consequently, the reactances of the lines that were less heavily loaded were decreased to increase the flow. However, there are cases with opposite adjustments and it is not straightforward to guess the direction of FACTS adjustment in meshed transmission networks. This is exactly why algorithms like the one presented in this paper are needed.

To ensure that the method is robust against the placement of the FACTS devices, various other factors are considered to determine the FACTS locations. Many factors, such as longterm load patterns and fuel prices, play a role in the FACTS planning problem. In order to show the effectiveness of our method, several scenarios for which the location of the devices is chosen based on other factors (e.g., reactance, line utilization, and capacity) are simulated. Tables IV to VII show the cost and savings as a percentage of the maximum possible savings (transportation model) using our two-stage linear method. The FACTS devices are located on the lines with highest reactance for the results shown in Table IV. The table presents the cost and savings when 5, 10, 15, and 20 devices are installed in the system. Our method took an average of 18 milliseconds, while MILP took an average of 822 milliseconds for each of the simulations.

TABLE III

PERCENTAGE CHANGE IN THE REACTANCE OF THE LINES UNDER DIFFERENT SCENARIOS

\begin{tabular}{|c|c|c|c|c|c|c|c|}
\hline \multirow[t]{2}{*}{ Line } & \multirow{2}{*}{$\begin{array}{l}\text { Loading } \\
(\%)\end{array}$} & \multicolumn{2}{|c|}{$\begin{array}{c}F A C T S C a- \\
\text { pacity }(\%)\end{array}$} & \multirow[t]{2}{*}{ Line } & \multirow{2}{*}{$\begin{array}{l}\text { Loading } \\
(\%)\end{array}$} & \multicolumn{2}{|c|}{$\begin{array}{c}F A C T S C a- \\
\text { pacity }(\%)\end{array}$} \\
\hline & & $70 \%$ & $90 \%$ & & & $70 \%$ & $90 \%$ \\
\hline $21-22$ & $22 \%$ & 70 & - & $69-75$ & $33 \%$ & -70 & - \\
\hline $23-24$ & $28 \%$ & -70 & - & $69-77$ & $97 \%$ & - & 28 \\
\hline $23-25$ & $50 \%$ & -70 & - & $70-74$ & $18 \%$ & -70 & - \\
\hline $24-70$ & $9 \%$ & -70 & -90 & $70-75$ & $14 \%$ & - & -90 \\
\hline $27-28$ & $18 \%$ & 70 & - & $75-77$ & $9 \%$ & -70 & -89 \\
\hline $30-17$ & $39 \%$ & -70 & - & $76-77$ & $9 \%$ & - & 90 \\
\hline $38-37$ & $36 \%$ & -70 & - & $77-82$ & $100 \%$ & 70 & 82 \\
\hline $38-65$ & $18 \%$ & -70 & - & $80-96$ & $55 \%$ & -30 & - \\
\hline $49-66$ & $27 \%$ & - & -84 & $80-98$ & $43 \%$ & -64 & - \\
\hline $49-69$ & $22 \%$ & 70 & - & $80-99$ & $39 \%$ & - & -57 \\
\hline $54-59$ & $6 \%$ & -70 & - & $81-80$ & $72 \%$ & 52 & 90 \\
\hline $56-59$ & $6 \%$ & -70 & - & $82-83$ & $82 \%$ & 70 & - \\
\hline $65-66$ & $26 \%$ & 70 & - & $85-88$ & $45 \%$ & -70 & - \\
\hline $68-81$ & $72 \%$ & - & 90 & $89-92$ & $100 \%$ & 34 & - \\
\hline $69-70$ & $24 \%$ & -25 & - & $91-92$ & $46 \%$ & -70 & -77 \\
\hline
\end{tabular}

Table $\mathrm{V}$ presents the same results for the case where the FACTS devices are installed on the lines with lowest reactances. The savings achieved by this placement policy are small. Our method took on average 17 milliseconds for each simulation, while MIP took on average 378 milliseconds. For 27 out of 32 cases presented in Table V, our algorithm was able to find the global optimal solution. For the five cases where the algorithm found a sub-optimal solution, the average distance to the global optimal solution was $\$ 0.47 / \mathrm{h}$ and the maximum distance was $\$ 1.5$. Table VI presents the cost and saving for the situation where the FACTS devices are placed on the lines with higher utilization. This case achieves the most savings among the four placement policies simulated for IEEE 118 bus system in this paper. The results show that only few mid-size FACTS devices can result in significant savings. The average computational time for our two-stage LP method was 18 milliseconds, while MIP took on average 326 milliseconds to find the solution.

Similar results are shown in Table VII for the case where FACTS devices are located on the lines with larger capacities. The number of devices installed in this case is $2,3,8$, and 27. For all but one of the cases presented in this table, our algorithm was able to find the global optimal solution. The average 
computational time for the two-stage LP was 18 milliseconds, while MILP took an average of 272 milliseconds to find the solution.

TABLE IV

COST REDUCTION COMPARED TO THE TRANSPORTATION NETWORK IF THE FACTS DEVICES ARE INSTALLED ON THE LINES WITH LARGER REACTANCES

\begin{tabular}{|c|c|c|c|c|c|c|c|c|}
\hline \multirow{3}{*}{$\begin{array}{c}\text { FACTS } \\
\text { Cap. }\end{array}$} & \multicolumn{8}{|c|}{ Number of FACTS devices } \\
\hline & \multicolumn{2}{|r|}{5} & \multicolumn{2}{|r|}{10} & \multicolumn{2}{|r|}{15} & \multicolumn{2}{|r|}{20} \\
\hline & $\begin{array}{l}\text { Cost } \\
(\$ / \mathrm{h}) \\
\end{array}$ & $\begin{array}{c}\text { Savings } \\
(\%)\end{array}$ & $\begin{array}{l}\text { Cost } \\
(\$ / \mathrm{h}) \\
\end{array}$ & $\begin{array}{c}\text { Savings } \\
(\%)\end{array}$ & $\begin{array}{l}\text { Cost } \\
(\$ / h) \\
\end{array}$ & $\begin{array}{c}\text { Savings } \\
(\%)\end{array}$ & $\begin{array}{l}\text { Cost } \\
(\$ / \mathrm{h}) \\
\end{array}$ & $\begin{array}{c}\text { Savings } \\
(\%)\end{array}$ \\
\hline $2 \%$ & 2074 & $0 \%$ & 2074 & $0 \%$ & 2074 & $0 \%$ & 2072 & $0 \%$ \\
\hline $5 \%$ & 2074 & $0 \%$ & 2074 & $0 \%$ & 2074 & $0 \%$ & 2069 & $1 \%$ \\
\hline $10 \%$ & 2074 & $0 \%$ & 2073 & $0 \%$ & 2073 & $0 \%$ & 2064 & $1 \%$ \\
\hline $20 \%$ & 2073 & $0 \%$ & 2071 & $0 \%$ & 2071 & $0 \%$ & 2052 & $3 \%$ \\
\hline $30 \%$ & 2072 & $0 \%$ & 2069 & $1 \%$ & 2069 & $1 \%$ & 2037 & $5 \%$ \\
\hline $50 \%$ & 2069 & $1 \%$ & 2063 & $1 \%$ & 2063 & $1 \%$ & 1998 & $10 \%$ \\
\hline $70 \%$ & 2063 & $1 \%$ & 2054 & $3 \%$ & 2056 & $2 \%$ & 1954 & $16 \%$ \\
\hline $90 \%$ & 2052 & $3 \%$ & 2051 & $3 \%$ & 2051 & $3 \%$ & 1914 & $21 \%$ \\
\hline
\end{tabular}

TABLE V

COST REDUCTION COMPARED TO THE TRANSPORTATION NETWORK IF THE FACTS DEVICES ARE INSTALLED ON THE LINES WITH SMALLER REACTANCE

\begin{tabular}{c|cc|cc|cc|cc}
\hline \multirow{2}{*}{$\begin{array}{c}\text { FACTS } \\
\text { Cap. }\end{array}$} & \multicolumn{2}{|c|}{5} & \multicolumn{9}{c}{ Number of FACTS devices } \\
\cline { 2 - 9 } & $\begin{array}{c}\text { Cost } \\
(\$ / \mathrm{h})\end{array}$ & $\begin{array}{c}\text { Savings } \\
(\%)\end{array}$ & $\begin{array}{c}\text { Cost } \\
(\$ / \mathrm{h})\end{array}$ & $\begin{array}{c}\text { Savings } \\
(\%)\end{array}$ & $\begin{array}{c}\text { Cost } \\
(\$ / \mathrm{h})\end{array}$ & $\begin{array}{c}\text { Savings } \\
(\%)\end{array}$ & $\begin{array}{c}\text { Cost } \\
(\$ / \mathrm{h})\end{array}$ & $\begin{array}{c}\text { Savings } \\
(\%)\end{array}$ \\
\hline $2 \%$ & 2074 & $0 \%$ & 2074 & $0 \%$ & 2074 & $0 \%$ & 2074 & $0 \%$ \\
$5 \%$ & 2074 & $0 \%$ & 2074 & $0 \%$ & 2073 & $0 \%$ & 2073 & $0 \%$ \\
$10 \%$ & 2074 & $0 \%$ & 2074 & $0 \%$ & 2071 & $0 \%$ & 2071 & $0 \%$ \\
$20 \%$ & 2074 & $0 \%$ & 2073 & $0 \%$ & 2068 & $1 \%$ & 2068 & $1 \%$ \\
$30 \%$ & 2074 & $0 \%$ & 2072 & $0 \%$ & 2065 & $1 \%$ & 2065 & $1 \%$ \\
$50 \%$ & 2074 & $0 \%$ & 2071 & $0 \%$ & 2059 & $2 \%$ & 2059 & $2 \%$ \\
$70 \%$ & 2074 & $0 \%$ & 2069 & $1 \%$ & 2052 & $3 \%$ & 2052 & $3 \%$ \\
$90 \%$ & 2074 & $0 \%$ & 2067 & $1 \%$ & 2045 & $4 \%$ & 2045 & $4 \%$ \\
\hline
\end{tabular}

TABLE VI

COST REDUCTION COMPARED TO THE TRANSPORTATION NETWORK IF THE FACTS DEVICES ARE INSTALLED ON THE LINES WITH HIGHER UTILIZATION

\begin{tabular}{|c|c|c|c|c|c|c|c|c|}
\hline \multirow{3}{*}{$\begin{array}{c}\text { FACTS } \\
\text { Cap. }\end{array}$} & \multicolumn{8}{|c|}{ Number of FACTS devices } \\
\hline & \multicolumn{2}{|r|}{5} & \multicolumn{2}{|r|}{10} & \multicolumn{2}{|c|}{15} & \multicolumn{2}{|r|}{20} \\
\hline & $\begin{array}{l}\text { Cost } \\
(\$ / \mathrm{h})\end{array}$ & $\begin{array}{c}\text { Savings } \\
(\%)\end{array}$ & $\begin{array}{l}\text { Cost } \\
(\$ / h)\end{array}$ & $\begin{array}{c}\text { Savings } \\
(\%)\end{array}$ & $\begin{array}{l}\text { Cost } \\
(\$ / h)\end{array}$ & $\begin{array}{c}\text { Savings } \\
(\%)\end{array}$ & $\begin{array}{l}\text { Cost } \\
(\$ / h)\end{array}$ & $\begin{array}{c}\text { Savings } \\
(\%)\end{array}$ \\
\hline $2 \%$ & 2047 & $4 \%$ & 2043 & $4 \%$ & 2032 & $5 \%$ & 2028 & $6 \%$ \\
\hline $5 \%$ & 2005 & $9 \%$ & 1995 & $10 \%$ & 1967 & $14 \%$ & 1957 & $15 \%$ \\
\hline $10 \%$ & 1935 & $18 \%$ & 1915 & $21 \%$ & 1857 & $28 \%$ & 1834 & $31 \%$ \\
\hline $20 \%$ & 1796 & $36 \%$ & 1753 & $42 \%$ & 1660 & $54 \%$ & 1637 & $57 \%$ \\
\hline $30 \%$ & 1669 & $53 \%$ & 1647 & $55 \%$ & 1590 & $63 \%$ & 1580 & $64 \%$ \\
\hline $50 \%$ & 1567 & $66 \%$ & 1521 & $72 \%$ & 1521 & $72 \%$ & 1499 & $75 \%$ \\
\hline $70 \%$ & 1566 & $66 \%$ & 1502 & $74 \%$ & 1501 & $74 \%$ & 1474 & $78 \%$ \\
\hline $90 \%$ & 1566 & $66 \%$ & 1485 & $76 \%$ & 1484 & $77 \%$ & 1450 & $81 \%$ \\
\hline
\end{tabular}

TABLE VII

COST REDUCTION COMPARED TO THE TRANSPORTATION NETWORK IF THE FACTS DEVICES ARE INSTALLED ON THE LINES WITH HIGHER CAPACITY

\begin{tabular}{|c|c|c|c|c|c|c|c|c|}
\hline \multirow{3}{*}{$\begin{array}{c}\text { FACT } \\
\text { S } \\
\text { Cap. }\end{array}$} & \multicolumn{8}{|c|}{ Number of FACTS devices } \\
\hline & \multicolumn{2}{|c|}{$>1.1 \mathrm{GVA}(2)$} & \multicolumn{2}{|c|}{$\begin{array}{l}>880 \text { MVA } \\
\text { (3) }\end{array}$} & \multicolumn{2}{|c|}{$\begin{array}{c}\text { >660 MVA } \\
(8)\end{array}$} & \multicolumn{2}{|c|}{$\begin{array}{c}>440 \text { MVA } \\
(27)\end{array}$} \\
\hline & $\begin{array}{l}\text { Cost } \\
(\$ / \mathrm{h}) \\
\end{array}$ & $\begin{array}{c}\text { Savings } \\
(\%)\end{array}$ & $\begin{array}{l}\text { Cost } \\
(\$ / h) \\
\end{array}$ & $\begin{array}{c}\text { Savings } \\
(\%)\end{array}$ & $\begin{array}{l}\text { Cost } \\
(\$ / h)\end{array}$ & $\begin{array}{c}\text { Savings } \\
(\%)\end{array}$ & $\begin{array}{l}\text { Cost } \\
(\$ / h) \\
\end{array}$ & $\begin{array}{c}\text { Savings } \\
(\%)\end{array}$ \\
\hline $2 \%$ & 2074 & $0 \%$ & 2074 & $0 \%$ & 2071 & $0 \%$ & 2067 & $1 \%$ \\
\hline $5 \%$ & 2074 & $0 \%$ & 2074 & $0 \%$ & 2065 & $1 \%$ & 2057 & $2 \%$ \\
\hline $10 \%$ & 2074 & $0 \%$ & 2074 & $0 \%$ & 2055 & $2 \%$ & 2039 & $5 \%$ \\
\hline $20 \%$ & 2074 & $0 \%$ & 2074 & $0 \%$ & 2036 & $5 \%$ & 2003 & $9 \%$ \\
\hline $30 \%$ & 2074 & $0 \%$ & 2074 & $0 \%$ & 2015 & $8 \%$ & 1967 & $14 \%$ \\
\hline $50 \%$ & 2074 & $0 \%$ & 2074 & $0 \%$ & 1972 & $13 \%$ & 1892 & $24 \%$ \\
\hline $70 \%$ & 2074 & $0 \%$ & 2074 & $0 \%$ & 1926 & $19 \%$ & 1815 & $34 \%$ \\
\hline $90 \%$ & 2074 & $0 \%$ & 2074 & $0 \%$ & 1876 & $26 \%$ & 1768 & $40 \%$ \\
\hline
\end{tabular}

Tables IV-VII include 128 simulated cases. For all but six of these cases, the solution obtained by our two-stage LP method was equivalent to the solution obtained by MILP; such empirical evidence suggests that this technique is highly likely to obtain the optimal solution. Again, this confirms the insight that was presented in the introduction: it is unlikely that key transmission bottlenecks would have a reverse in the power flow direction as a result of the optimal FACTS operation. For the cases, where the solution obtained by our method was suboptimal, it was within a very small epsilon distance from the optimal solution.

\section{B. Polish System - Winter 2000 Morning Peak}

The test case data is taken from [49]-[50]. The system consists of 2383 buses, 2896 transmission lines, and 327 generators. The difference between the cost obtained by DCOPF and transportation model is $\$ 30,886$, which is the maximum savings that can be achieved via flow control technologies. Table VIII shows the savings for the case, where different size FACTS devices are installed on the lines with larger reactances. The average solution time for our two-stage LP method was $569 \mathrm{~ms}$ with a standard deviation of $39 \mathrm{~ms}$, while average solution time for MILP formulation was $2783 \mathrm{~ms}$ with a standard deviation of $1254 \mathrm{~ms}$. Table IX shows the same results for the case that FACTS devices are installed on the lines that are more heavily utilized. The average solution time for our two-stage LP method was $526 \mathrm{~ms}$ with a standard deviation of $34 \mathrm{~ms}$, while the average solution time for MILP formulation was $2331 \mathrm{~ms}$ with a standard deviation of $996 \mathrm{~ms}$. For all the 64 simulation studies on the Polish system, our two-stage LP algorithm was able to find the global optimal solution. The results confirm the effectiveness of our method both in its capability to find quality solutions and computational time reduction.

TABLE VIII

COST REDUCTION COMPARED TO THE TRANSPORTATION NETWORK IF THE FACTS DEVICES ARE INSTALLED ON THE LINES WITH LARGER REACTANCES

\begin{tabular}{|c|c|c|c|c|c|c|c|c|}
\hline \multirow{3}{*}{$\begin{array}{c}\text { FACTS } \\
\text { Cap. }\end{array}$} & \multicolumn{8}{|c|}{ Number of FACTS devices } \\
\hline & \multicolumn{2}{|l|}{5} & \multicolumn{2}{|l|}{10} & \multicolumn{2}{|l|}{15} & \multicolumn{2}{|l|}{20} \\
\hline & (\$) & (\%) & (\$) & $(\%)$ & (\$) & $(\%)$ & (\$) & $(\%)$ \\
\hline $2 \%$ & 136 & 0 & 184 & 1 & 224 & 1 & 253 & 1 \\
\hline $5 \%$ & 344 & 1 & 463 & 2 & 559 & 2 & 632 & 2 \\
\hline $10 \%$ & 702 & 2 & 930 & 3 & 1123 & 4 & 1270 & 4 \\
\hline $20 \%$ & 1466 & 5 & 1894 & 6 & 2214 & 7 & 2512 & 8 \\
\hline $30 \%$ & 2312 & 7 & 2909 & 9 & 3406 & 11 & 3870 & 13 \\
\hline $50 \%$ & 4410 & 14 & 5426 & 18 & 6067 & 20 & 6897 & 22 \\
\hline $70 \%$ & 7046 & 23 & 7833 & 25 & 9191 & 30 & 9895 & 32 \\
\hline $90 \%$ & 8977 & 29 & 9326 & 30 & 11289 & 37 & 11941 & 39 \\
\hline
\end{tabular}

TABLE IX

COST REDUCTION COMPARED TO THE TRANSPORTATION NETWORK IF THE FACTS DEVICES ARE INSTALLED ON THE LINES WITH HIGHER UTILIZATION

\begin{tabular}{r|rr|rr|rr|rr}
\hline \multirow{2}{*}{$\begin{array}{c}\text { FACTS } \\
\text { Cap. }\end{array}$} & \multicolumn{7}{|c}{ Number of FACTS devices } \\
& \multicolumn{1}{|c}{5} & \multicolumn{1}{c}{10} & \multicolumn{2}{c}{15} & \\
\cline { 2 - 8 } & \multicolumn{1}{|c}{$(\%)$} & $(\%)$ & $(\$)$ & $(\%)$ & $(\$)$ & $(\%)$ \\
\hline $2 \%$ & 280 & 1 & 955 & 3 & 1438 & 5 & 1563 & 5 \\
$5 \%$ & 701 & 2 & 2411 & 8 & 3615 & 12 & 3921 & 13 \\
$10 \%$ & 1403 & 5 & 4771 & 15 & 6858 & 22 & 7425 & 24 \\
$20 \%$ & 2813 & 9 & 8362 & 27 & 11631 & 38 & 12777 & 41 \\
$30 \%$ & 4212 & 14 & 11528 & 37 & 16534 & 54 & 17925 & 58 \\
$50 \%$ & 5769 & 19 & 18262 & 59 & 20967 & 68 & 22769 & 74 \\
$70 \%$ & 7227 & 23 & 21371 & 69 & 22569 & 73 & 24777 & 80 \\
$90 \%$ & 8898 & 29 & 22482 & 73 & 23388 & 76 & 25325 & 82 \\
\hline
\end{tabular}




\section{FutUre WORK AND NEXT STEPS}

Previous sections showed the effectiveness of a two-stage LP method for enhanced operation of FACTS devices. The paper aimed at serving as the proof of concept in a DC model with a relatively small test case. Although the results are promising, the following steps would be necessary to confirm the effectiveness of the method under more realistic set of assumptions:

- AC feasibility: AC feasibility of FACTS adjustments should be studied and confirmed before the solution can be implemented. In order to do that, further research is needed to include an AC model to check the solutions coming out of a DCOPF. Currently, AC feasibility is checked for and achieved via out-ofmarket adjustments [44]. Thus, depending on the application, the check or modification to the FACTS set point can also be done out-of-market to ensure AC feasibility.

- Stability: Similar to AC feasibility, the adjustment to the FACTS settings should be checked for dynamic stability before it can be implemented.

- Reliability: The results shown in this paper were not necessarily $\mathrm{N}-1$ reliable. In order to ensure reliability, a security assessment can be conducted to determine if the solutions are reliable and, if not, the SCED can be re-solved with appropriate cuts representing the post-contingency violations. Similar to transmission switching, FACTS devices can reduce the cost and improve reliability at the same time. Moreover, changing FACTS setting can be seen as a corrective instrument to ensure reliability. For instance, ongoing work is focused on incorporating this approach within real-time contingency analysis in order to use FACTS devices for corrective actions.

- Test on large-scale systems: Although the results were promising for the IEEE 118 bus and the Polish system, the method should be tested on larger-scale systems to obtain better understanding of its performance in real world applications. More testing on large-scale systems will further demonstrate the benefit of this approach in regards to scalability.

- Impacts of reactance control on current markets and operational procedures: Changing the lines' reactances would influence other markets such as financial transmission rights (FTR) markets. Transmission switching has been shown to cause revenue inadequacy in FTR markets [51]. Similar studies are needed for FACTS devices. FACTS adjustments would also affect the settings of protection relays as well as affect state estimation. Therefore, the FACTS settings should be incorporated into state estimation and protection systems should be adjusted when substantial changes in the line impedance occur. These impacts need to be studied in detail. One potential solution regarding protection systems is to use settingless protection systems.

This paper only showed the reformulation of FACTS setting adjustment to a two-stage LP for economic benefits. In order to exploit the full benefits of this method, further re- search is needed:

- Inclusion of FACTS in different stages of the market: the flexibility that FACTS offers can be used at all stages of the market. Particularly, it should be included in day-ahead SCUC. FACTS settings can also be adjusted in hour-ahead and real-time markets for reliability and cost saving purposes.

- FACTS adjustment as a corrective instrument: The flexibility that FACTS devices offer can be used as corrective actions in a post-contingency state to enhance deliverability of reserve. The two-stage LP formulation can provide fast corrective options for the operator in real time.

- $\quad$ AC model: The formulation provided in this paper was based on a DCOPF. As was stated previously in this paper, most of the market procedures today are done using a DC model. Therefore, economic adjustment of FACTS settings in day-ahead and real-time markets based on a DC model seems to be consistent with the current practices of the industry. However, AC based modeling of FACTS adjustment is also valuable, e.g., for real-time contingency analysis where FACTS can be used to perform corrective actions.

\section{CONCLUSIONS}

The transmission system in the US is under stress and needs to be upgraded. A cheaper and more efficient alternative to building new transmission lines would be better utilization of the current network. This paper showed how inclusion of reactance adjustment by FACTS devices in an OPF formulation can support a lower cost solution. It was shown that such formulation would result in an NLP. The NLP was reformulated to a MILP. Then, the MILP was converted to a two-stage LP. The solution to this two-stage LP is equivalent to the solution obtained by the original MILP, under the condition that the sign of voltage angle differences, on the lines equipped with FACTS, do not change in the optimal solution. The simulation studies show that the method was able to find the optimal solution for all but 8 out of 448 cases on IEEE 118 bus system, and all the 64 cases on the Polish system that were simulated in this paper. For the cases, where optimal solution was not found, the difference between the cost achieved by the two-stage LP method and the original MILP was very small. The method was able to find the solution much faster than the original MILP. Although there is no guarantee our two-stage LP always finds the optimal solution, significant savings could be achieved using the proxy linear formulation developed in this paper within a reasonable time. Further research is needed to improve the model and show its effective with more realistic test cases.

\section{REFERENCES}

[1] US Energy Information Administration, "Electric power annual 2012," US Department of Energy, Dec. 2013, [Online]. Available: http://www.eia.gov/electricity/annual/pdf/epa.pdf.

[2] A. Spencer, "National transmission grid study," US Department of Energy, 2002, [Online]. Available: http://www.ferc.gov/industries/electric/gen-info/transmission-grid.pdf. 
[3] S. W. Snarr, "The commerce clause and transmission infrastructure development: an answer to jurisdictional issues clouded by protections," Electr. J., vol. 22, no. 5, pp. 8-18, June 2009.

[4] Federal Energy Regulatory Commission, "Order No. 1000 - transmission planning and cost Allocation," Docket No. RM10-23-000., 2011, [Online]. Available: http://www.ferc.gov/industries/electric/indusact/trans-plan.asp.

[5] S. M. Amin, "Securing the electricity grid," NAE The Bridge, vol. 40, no. 1, pp. 13-20, Spring 2010.

[6] E. B. Fisher, R. P. O'Neill, and M. C. Ferris, "Optimal transmission switching," IEEE Trans. Power Syst., vol. 23, no. 3, pp. 1346-1355, Aug. 2008

[7] K. W. Hedman, R. P. O'Neill, E. B. Fisher, and S. S. Oren, "Optimal transmission switching-sensitivity analysis and extensions," IEEE Trans. Power Syst., vol. 23, no. 3, pp. 1469-1479, Aug. 2008.

[8] K. W. Hedman, R. P. O’Neill, E. B. Fisher, and S. S. Oren, "Optimal transmission switching with contingency analysis," IEEE Trans. Power Syst., vol. 24, no. 3, pp. 1577-1586, Aug. 2009.

[9] K. W. Hedman, M. C. Ferris, R. P. O’Neill, E. B Fisher, and S. S. Oren, "Co-optimization of generation unit commitment and transmission switching with N-1 reliability," IEEE Trans. Power Syst., vol. 25, no. 2, pp. 1052-1063, May 2010

[10] C. Lehmkoster, "Security constrained optimal power flow for an economical operation of FACTS devices in liberalized energy markets," IEEE Trans. Power Del., vol. 17, no. 2, pp. 603-608, Apr. 2002.

[11] G. N. Taranto, L. M. V. G. Pinto, and M. V. F. Pereira, "Representation of FACTS devices in power system economic dispatch," IEEE Trans. Power Syst., vol. 7, no. 2, May 1992.

[12] C. Barrows and S. Blumsack, "Transmission switching in the RTS-96 test system," IEEE Trans. Power Syst., vol. 276, no. 2, pp. 1134-1135, May 2012.

[13] A. S. Korad and K. W. Hedman, "Robust corrective topology control for system reliability," IEEE Trans. Power Syst., vol. 28, no. 4, pp. 4042 4051, Nov. 2013.

[14] C. Lueken, P. Carvalho, and J. Apt, "Distribution grid reconfiguration reduces power losses and helps integrate renewables," Energ. Policy, vol. 48, pp. 260-273, Sep. 2012.

[15] P. Balasubramanian and K. W. Hedman, "Real-time corrective switching in response to simultaneous contingencies," J. Energy Eng., 2014.

[16] M. A. Khorsand and K. W. Hedman, "Day-ahead corrective transmission topology control," in Proc. IEEE PES General Meeting, July 2014.

[17] W. Shao and V. Vittal, "Corrective switching algorithm for relieving overloads and voltage violations," IEEE Trans. Power Syst., vol. 20, no. 4, pp. 1877-1885, Nov. 2005.

[18] M. Sahraei-Ardakani, A. Korad, K. W. Hedman, P. Lipka, and S. Oren, "Performance of AC and DC based transmission switching heuristics on a large-scale polish system," in Proc. IEEE PES General Meeting, July 2014

[19] T. Potluri and K. W. Hedman, "Impacts of topology control on the ACOPF," in Proc. IEEE PES General Meeting, July 2012.

[20] G. M. Huang, W. Wang, and J. An, "Stability issues of smart grid transmission line switching," in Proc. of 19th IFAC World Congress, pp. 24-29, Aug. 2014.

[21] N. G. Hingorani and L. Gyugyi, Understanding FACTS: concepts and technology of flexible AC transmission systems, Wiley-IEEE Press, Dec. 1999.

[22] P. Fairley, "Flexible AC transmission: the FACTS machine," IEEE Spectrum Magazine, Jan. 2011.

[23] Smart Wire Grid, [Online]. Available: http://www.smartwiregrid.com/technology/.

[24] J. Hauer, T. Overbye, J. Dagle, and S. Widergren, "Advanced transmission technologies," National Transmission Grid Study, 2002, [Online]. Available: http://certs.lbl.gov/ntgs/issue-6.pdf.

[25] Y. Xiao, Y. H. Song, C. C. Liu, and Y. Z. Sun, "Available transfer capability enhancement using FACTS devices," IEEE Trans. Power Syst., vol. 18, no. 1, pp. 305-312, Feb. 2003.

[26] H. Mori and Y. Maeda, "Application of two-layered tabu search to optimal allocation of UPFC for maximizing transmission capability," IEEE Intl. Symp. Circ. Syst., 2006.

[27] T. Orfanogianni and R. Bacher, "Steady-state optimization in power systems with series FACTS devices," IEEE Trans. Power Syst., vol. 18, no. 1, pp. 19-26, Feb. 2003.

[28] C. C. Liu, G. T. Heydt, and A. A. Edris, "Impact of FACTS controllers on transfer capability of power grids," IEEE PES Winter Meeting, 2002.
[29] M. Amin, "North American electricity infrastructure: system security, quality, reliability, availability, and efficiency challenges and their societal impacts," Continuing Crises in National Transmission Infrastructure: Impacts and Options for Modernization. National Science Foundation (NSF), 2004.

[30] ISO New England, "Regional system plan 2014," ISO-NE, 2014.

[31] S. Basler, S. Sankar, R. Miller, M. Israel, T. Curry, and T. Mason, "Effective grid utilization: a technical assessment and application guideline". National Renewable Energy Laboratory, 2012.

[32] Federal Energy Regulatory Commission, "Order on petition for declaratory order and related determinations," Docket No: ER10-253-000, EL10-14-000, Apr. 2010

[33] S. Cvijić and M. D. Ilić, "Part I: a new framework for modeling and tracing of bilateral transactions and the corresponding loop flows in multi-control area power networks," IEEE Trans. Power Syst., vol. 29, no. 6, pp. 2706-2714, Nov. 2014.

[34] S. Cvijić and M. D. Ilić, "Part II: PAR flow control based on the framework for modeling and tracing of bilateral transactions and corresponding loop flows," IEEE Trans. Power Syst., vol. 29, no. 6, pp. 2715-2722, Nov. 2014.

[35] J. Mohammadi, G. Hug, and S. Kar, “A Benders' decomposition approach to corrective security constrained OPF with power flow control devices," in Proc. IEEE PES General Meeting, July 2013.

[36] G. Hug, "Generation cost and system risk trade-off with corrective power flow control," in Proc. 50 $0^{\text {th }}$ Annu. Allerton Conf., pp. 1324-1333, Oct. 2012.

[37] J. Mohammadi, G. Hug, and S. Kar, "On the behavior of responsive loads in the presence of DFACTS devices," in Proc. North American Power Symp., Sept. 2012.

[38] G. Hug, "Coordinated power flow control to enhance steady state security in power systems," Ph.D. Dissertation, Swiss Federal Institute of Technology, Zurich, 2008.

[39] J. Cardell, "A real time price signal for FACTS devices to reduce transmission congestion," in Proc. of $40^{\text {th }}$ HICSS, 2007.

[40] R. P. O'Neill, E. B. Fisher, B. F. Hobbs, and R. Baldick, "Towards a complete real-time electricity market design," J. Reg. Econ., vol. 34, pp. 220-250, May 2008

[41] M. Sahraei-Ardakani and S. Blumsack, "Market equilibrium for dispatchable transmission using FACT devices," in Proc. IEEE PES General Meeting, July 2012.

[42] M. Sahraei-Ardakani and S. Blumsack, "Marginal value of FACTS devices in transmission-constrained electricity markets," in Proc. IEEE PES General Meeting, July 2013.

[43] R. Yang and G. Hug, "Regression-based control of thyristor-controlled series compensators for optimal usage of transmission capacity," IET Gener. Transm. Distrib., vol. 8, no. 8, pp. 1444-1452, Aug. 2014.

[44] Y. M. Al-Abdullah, M. Abdi-Khorsand, and K. W. Hedman, "The role of out-of-market corrections in day-ahead scheduling," IEEE Trans. Power Syst., accepted for publication.

[45] Dept. Electr. Eng., Univ. Washington, "Power system test case archive," 2007 [Online]. Available: http://www.ee.washington.edu/research/.

[46] S. A. Blumsack, "Network topologies and transmission investment, under electric-industry restructuring," Ph.D. dissertation, Eng. Public Pol., Carnegie Mellon Univ., Pittsburgh, PA, 2006.

[47] M. Sahraei-Ardakani, "IEEE 118-bus test System," [Online]. Available: http://www.public.asu.edu/ msahraei/ieee118.

[48] P. A. Ruiz, J. M. Foster, A. Rudkevich, and M. C. Caramanis, "Tractable transmission topology control using sensitivity analysis," IEEE Trans. Power Syst., vol. 27, no. 3, pp. 1550-1559, Aug. 2012.

[49] R. D. Zimmerman, C. E. Murillo-Sánchez, and R. J. Thomas, "MATPOWER: Steady-State Operations, Planning and Analysis Tools for Power Systems Research and Education," Power Systems, IEEE Transactions on, vol. 26, no. 1, pp. 12-19, Feb. 2011.

[50] R. D. Zimmerman, C. E. Murillo-Sánchez, and D. Gan, MATPOWER, [Online]. Available: http://www.pserc.cornell.edu/matpower/

[51] K. Hedman, S. Oren, and R. P. O'Neill. "Optimal transmission switching: economic efficiency and market implications." J. Reg. Econ., vol. 40, no. 2, pp. 111-140, June 2011 
Mostafa Sahraei-Ardakani (M'06) received the $\mathrm{PhD}$ degree in energy engineering from The Pennsylvania State University, University Park, PA in 2013. $\mathrm{He}$ also holds the B.S. and M.S. degrees in electrical engineering from University of Tehran, Iran. Currently, he is a post-doctoral scholar in the School Electrical, Computer and Energy Engineering at Arizona State University. His research interests include energy economics and policy, electricity markets, power system optimization, transmission network, and the smart grid.

Kory W. Hedman (S' 05, M' 10) received the B.S. degree in electrical engineering and the B.S. degree in economics from the University of Washington, Seattle, in 2004 and the M.S. degree in economics and the M.S. degree in electrical engineering from Iowa State University, Ames, in 2006 and 2007, respectively. He received the M.S. and Ph.D. degrees in industrial engineering and operations research from the University of California, Berkeley in 2008 and 2010 respectively.

Currently, he is an assistant professor in the School of Electrical, Computer, and Energy Engineering at Arizona State University. He previously worked for the California ISO (CAISO), Folsom, CA, on transmission planning and he has worked with the Federal Energy Regulatory Commission (FERC), Washington, DC, on transmission switching. His research interests include power systems operations and planning, electricity markets, power systems economics, renewable energy, and operations research. 